\title{
ARTICLE The mGluR2/3 agonist pomaglumetad methionil normalizes aberrant dopamine neuron activity via action in the ventral hippocampus
}

\author{
Susan F. Sonnenschein ${ }^{1}$ and Anthony A. Grace ${ }^{1}$
}

\begin{abstract}
The group 2 metabotropic glutamate receptor (mGluR2/3) agonist, pomaglumetad methionil (POM), showed promise as a novel antipsychotic in preclinical research but failed to show efficacy in clinical trials, though it has been suggested that it may be effective in certain patient populations. Although previous studies have shown that mGluR2/3 agonists have no effect on dopamine (DA) in wild type rats, we used the methylzoxymethanol acetate (MAM) model to determine whether POM may indirectly normalize DA neuron activity in a model representative of the hyperdopaminergic state thought to underlie psychosis, compared to SAL rats, using in vivo, anesthetized, electrophysiological recordings. POM dose-dependently reduced the number of spontaneously active DA neurons in the VTA of MAM rats to control levels without affecting DA firing in SAL rats, which persisted following 14d repeated treatment with POM. In female MAM rats, POM significantly reduced DA neuron population activity only during proestrous and estrous stages. MAM rats also demonstrated dose-dependent improvement in novel object recognition following acute POM, which was not observed in SAL rats. Similar to the MAM rats, DA neuron population activity was increased in a hippocampal-dependent manner following acute restraint stress. Administration of POM prior to $2 \mathrm{~h}$ restraint stress prevented the restraint-induced increase in DA neuron population activity, and this effect was blocked by pretreatment with an mGluR2/3 antagonist. Thus, the ability of POM to reduce the hyperdopaminergic activity in both MAM rats and in wild type rats following restraint stress suggests that it can indirectly regulate DA neuron activity, which may underlie its potential therapeutic effects.
\end{abstract}

Neuropsychopharmacology (2020) 45:2106-2113; https://doi.org/10.1038/s41386-020-0764-2

\section{INTRODUCTION}

Antipsychotic drugs (APDs) that target dopamine (DA) $D_{2}$ receptors have remained the primary treatment for schizophrenia since their introduction in the 1950s [1]. Their therapeutic effect is attributed to $D_{2}$ receptor antagonist or partial agonist action [2-4] that reduces elevated DA neuron activity [5-7]. Dysregulated presynaptic DA neuron activity $[8,9]$ is observed in patients with schizophrenia and correlated to positive symptom severity (i.e. hallucinations, delusions, thought disorder). Accordingly, patients who respond to current APDs primarily display a reduction in positive symptoms [10]. However, $\sim 30 \%$ of patients with schizophrenia display treatment resistance with little or no response to initial treatment [11]. Even clozapine, the treatment of choice for refractory symptoms, is ineffective in $\sim 50 \%$ of patients who take it [12-14]. Current APDs are also hindered by a high rate of treatment nonadherence, driven by factors including limited efficacy and poor tolerability [15]. Furthermore, there is evidence of persistent and potentially detrimental changes to brain morphology $[16]$ and the DA system $[17,18]$ with long-term use of $D_{2}$ receptor antagonists. These shortcomings highlight the need for alternative strategies, including novel APD mechanisms.

One of the most promising novel target candidates are the group II metabotropic glutamate receptors, comprised of mGluR2 and mGluR3 (mGluR2/3). mGluR2/3 are prominently expressed in limbic brain regions, where they are primarily localized presynaptically on glutamatergic terminals to regulate excessive glutamate release [19]. Aberrant excitatory-inhibitory balance has been well-characterized in schizophrenia as an underlying component of numerous symptoms of the disorder [20, 21], including a primary driver of DA dysregulation [22]. Despite extensive preclinical support for mGluR2/3 as a novel APD target $[23,24]$ and success in an early clinical trial [25], the mGluR2/3 agonist pomaglumetad methionil (POM) was pulled from phase III clinical trials for insufficient efficacy in reducing psychotic symptoms compared to current APDs [26]. Later analyses found that POM was more effective in certain populations, including early-in-disease patients [27]. Thus, further research is necessary to clarify the disconnect between the preclinical research and clinical trial results.

The impact of mGluR2/3 agonists on DA neuron activity is critical to understanding its effects as a potential antipsychotic drug. Prior research on mGluR2/3 agonists primarily focused on the prefrontal cortex [28-31]. Their potential therapeutic effect was suggested to be DA-independent due absence of mGluR2/3 expression in the ventral tegmental area (VTA) [32-34] and their lack of effect on DA levels at baseline or following VTA stimulation [31]. However, mGluR2/3 is densely expressed in the hippocampus (HPC), including the human anterior HPC [35] and corresponding rat ventral HPC (vHPC) [33, 34]. The anterior HPC of patients with schizophrenia has been shown to demonstrate a loss of

${ }^{1}$ Departments of Neuroscience, Psychiatry and Psychology, Center for Neuroscience, University of Pittsburgh, Pittsburgh, PA 15260, USA

Correspondence: Susan F. Sonnenschein (sfs29@pitt.edu)

Received: 29 January 2020 Revised: 27 May 2020 Accepted: 8 July 2020

Published online: 14 July 2020 
parvalbumin positive $(\mathrm{PV}+)$ interneurons and increased measures of activity that correlates with psychosis, which may be a result of pyramidal neuron disinhibition [36, 37]. A similar progression of PV + interneuron loss [38] and VHPC hyperactivity [39] is observed in the methylazoxymethanol acetate (MAM) rodent model of schizophrenia. The increased VHPC drive results in an increased number of spontaneously active DA neurons in the VTA, which produces a hyperdopaminergic state consistent with the pathology observed in patients [40-42].

MAM rats thus provide a clinically relevant model to determine how mGluR2/3 activation may affect a system-wide pathophysiology and examine region-specific effects. mGluR2/3 agonists have been previously shown to reduce increased HPC activity in stressed wild type rats, based on cFos expression [43], and so we also examined role of $\mathrm{mGluR2} / 3$ activation on HPC function, based on a novel object recognition task (NOR). MAM rats have been shown previously to display reduced NOR compared to SAL rats [44], which may relate to impairment of HPC function. NOR is considered a working memory task when there is a short delay between the two trials. However, it has been shown to be HPCdependent when there is a long delay, such as the $1 \mathrm{~h}$ used between trials in the present task [45]. MAM rats provide a model of consistently increased VHPC activity, though in a normal system, the VHPC regulates DA neuron activity in a contextdependent manner, such that increased vHPC activity results in a transient increase DA neuron activity in a normal system in a context-dependent manner, as previously shown with restraintstress [46]. Therefore, we also examined whether mGluR2/3 activation can prevent the transient restraint-stress induced increase in DA neuron population activity in wild type rats. Our overall aim was to determine whether the mGluR2/3 agonist, POM, may regulate DA neuron activity in states of increased $\mathrm{vHPC}$ activity and our findings demonstrate that mGluR2/3 agonists act on the hyperactive vHPC to indirectly reduce increased DA neuron population activity in the VTA and improve NOR performance.

\section{METHODS}

Subjects

All procedures were conducted in accordance with the National Institute of Health Guide for the Care and Use of Laboratory Animals and approved by the University of Pittsburgh Institutional Animal Care and Use Committee. Timed pregnant SpragueDawley dams (Envigo, Indianapolis, IN) were obtained on gestational day (GD) 15 and MAM $(20 \mathrm{mg} / \mathrm{kg}$, i.p., Midwest Research Institute, Kansas City, MO) or saline (SAL; $1 \mathrm{ml} / \mathrm{kg}$, i.p.) was administered on GD17. Male and female pups were weaned on postnatal day (PD)23. Naïve adult male Sprague-Dawley rats were obtained from Envigo for restraint stress experiments. All rats were housed in groups of 2-3 with littermates in a temperature $\left(22^{\circ} \mathrm{C}\right)$ and humidity (47\%)-controlled facility with ad libitum food and water in a normal 12-h light-dark cycle.

\section{Drug Administration}

LY2140023 (pomaglumetad methionil, POM), the prodrug of mGluR2/3 agonist LY404039, was obtained from Selleck Chemicals, Houston, TX. The mGluR2/3 antagonist LY341495 (LY34) was obtained from Tocris Bioscience, Minneapolis, MN. Systemic drug doses were based on previous preclinical studies performed with the drugs $[23,24,47]$ and microinfusion dose was determined from studies performed with the mGluR2/3 agonist LY379268 $[48,49]$. Both drugs were dissolved in $0.9 \%$ sterile saline and prepared at a volume of $1 \mathrm{ml} / \mathrm{kg}$, except the $10 \mathrm{mg} / \mathrm{kg}$ dose of POM, which was mixed at a volume of $3 \mathrm{ml} / \mathrm{kg}$ for improved solubility. Drugs were dissolved with dropwise addition of $1 \mathrm{M}$ $\mathrm{NaOH}$ to POM (pH 7) and LY34 (pH $~ 8)$ solutions. Vehicle (VEH)treated rats received $0.9 \%$ sterile saline. All systemic treatments were administered intraperitonially.
For electrophysiological experiments, POM or vehicle was administered to adult MAM and SAL rats following anesthetization and $30 \mathrm{~min}$ prior to electrophysiological recording. Recordings were performed within approximately $3-4 \mathrm{~h}$ of administration. For female rat recordings, vaginal lavages were taken following anesthetization. Estrous stage was determined using a light microscope based on criteria described in [50,51]. To examine the effects of repeated POM administration on DA neuron activity, adult male MAM and SAL rats were randomly assigned to either acute or repeated administration groups and received either acute or $14 \mathrm{~d}$ repeated daily i.p. administration of either POM or vehicle. On the $15^{\text {th }}$ day, rats received a final treatment $30 \mathrm{~min}$ prior to electrophysiological recording.

For acute, intra-vHPC microinfusions, rats were implanted ipsilateral to the side of the recording with a 23-gauge guide cannula $1.0 \mathrm{~mm}$ dorsal to the ventral subiculum or ventral CA1 at $-5.7 \mathrm{~mm}$ posterior and $+4.6 \mathrm{~mm}$ lateral from bregma, and -6.0 $\mathrm{mm}$ ventral from the top of skull. $0.5 \mu \mathrm{g}$ of POM was gradually infused over $2 \mathrm{~min}$ at a volume of $0.5 \mu \mathrm{l}$ through a 33-gauge injection cannula protruding $1.0 \mathrm{~mm}$ past the end of the implanted guide cannula. The injection cannula was left in place for 1 minute to ensure diffusion of drug into the surrounding tissue. The control group consisted of rats that received vehicle infusion into the vHPC. Recordings took place until $\sim 2-3 \mathrm{~h}$ after infusion.

\section{Electrophysiological recordings}

The activity state of DA neurons in the VTA was measured using in vivo extracellular recordings. Rats were anesthetized with chloral hydrate $(400 \mathrm{mg} / \mathrm{kg}$; i.p.) and placed on a stereotaxic frame (Kopf, Tujunga, CA). Supplemental anesthesia was administered i. p. to maintain suppression of the hind limb withdrawal reflex. Body temperature was maintained at $37^{\circ} \mathrm{C}$ with a temperaturecontrolled heating pad (CWE Inc., Ardmore, PA). Single-barrel glass electrodes (WPI, Sarasota, FL) were pulled vertically (PE-2, Narasige, Japan), broken under a microscope to an impedance of 6-8 $\mathrm{M} \Omega$, and filled with $2 \mathrm{M} \mathrm{NaCl}$ containing $2 \%$ Chicago Sky Blue dye in $2 \mathrm{M}$ saline. Electrodes were lowered with a hydraulic micropositioner (Kopf) to sample the VTA: AP: -5.3 to $-5.7 \mathrm{~mm}$ and ML: 0.6 to $1.0 \mathrm{~mm}$ from bregma, DV: -6.5 to $-9.0 \mathrm{~mm}$ from the top of brain). Recordings were performed by making 6-9 vertical electrode passes ("tracks") in a predetermined grid pattern with each track separated by $0.2 \mathrm{~mm}$. Single-unit activity was obtained using an amplifier (Fintronics, Orange, CT) using a highpass filter at $30 \mathrm{~Hz}$ and lowpass at $16 \mathrm{kHz}$. DA neurons were classified based on established criteria, including a biphasic action potential with duration $>2.2 \mathrm{~ms}, 1-10 \mathrm{~Hz}$ firing rate, and irregular and burst firing patterns with burst initiation defined as an interspike interval of $\leq 80 \mathrm{~ms}$ and termination as $>160 \mathrm{~ms}$ [52-54]. The activity of each DA neuron was recorded for at least $1 \mathrm{~min}$ of stable spontaneous activity using LabChart software (AD Instruments, Colorado Springs, CO).

At the end of each recording, electrode placement was verified following each experiment via electrophoretic ejection of Chicago Sky Blue dye from the tip of the recording electrode $(-20 \mu \mathrm{A}$ constant current, $20 \mathrm{~min}$ ). Rats were then overdosed with chloral hydrate and decapitated. The brains were removed and fixed for at least 48 hours ( $8 \%$ paraformaldehyde in PBS), cryoprotected (25\% sucrose in PBS) until saturated, and sliced on a cryostat into $60 \mu \mathrm{m}$ sections, which were mounted onto gelatin-coated slides. Slides were stained with a mixture of cresyl violet and neutral red for verification of electrode sites with reference to a stereotaxic atlas [55].

Electrophysiological recording analysis. Three parameters were analyzed for DA neuron activity: (1) the average number of spontaneously active DA neurons encountered per electrode track ("population activity"), (2) average firing rate and (3) the 
percentage of spikes that occurred in bursts (\%SIB). Analysis of firing rate and bursting activity was performed using NeuroExplorer (Plexon, Dallas, TX). Population activity was averaged within each animal and then across animals in each group, whereas the firing rate and burst activity of each neuron was counted as an independent replicate and averaged across animals in a group. Significance was assessed with a two-way ANOVA (MAM $\times$ Treatment or Condition $\times$ Treatment) followed by Tukey post hoc comparisons using GraphPad Prism 8 (GraphPad Software, San Diego, CA). Three-way ANOVA (MAM $\times$ Treatment $\times$ Time) was completed with SAS (SAS Institute, Cary, NC) to examine effects of acute vs repeated treatment.

\section{Novel object recognition}

The NOR test was conducted in a rectangular test box ( $L 70 \times$ W40 $\times \mathrm{H} 30 \mathrm{~cm}$ ). Each rat was habituated to the box for $10 \mathrm{~min}$ 1 day prior to the test. The test day involved two 5-min trials separated by a $1 \mathrm{~h}$ intertrial interval. Rats received a randomized injection of either VEH $(1 \mathrm{ml} / \mathrm{kg})$ or POM $(1 \mathrm{mg} / \mathrm{kg}$ or $3 \mathrm{mg} / \mathrm{kg})$, i.p. $30 \mathrm{~min}$ prior to the first trial. In the first trial (T1), rats were placed in the box containing two identical objects (glass bottles, L6.5 $\times$ W6.5 $\times \mathrm{H} 17.5$ ) that were each placed $\sim 20 \mathrm{~cm}$ from the far walls of the box. In the second trial (T2), one of the objects presented in T1 was replaced by a novel object (resin ornament, L10.5 $\times$ W5.0 $\mathrm{H} 6.5 \mathrm{~cm}$ ). The familiar and novel objects were too heavy to be displaced by the animals and had different shape, color and texture. The box and the objects were cleaned between each trial. Habituation and behavioral tests were performed during the dark cycle. The behavior was recorded on video and object interaction was scored by an experimenter blinded to treatment group. Interaction time (s) of each object in each trial was recorded manually by the use of stopwatches and defined as time when the rat interacts directly with the object, such as licking, sniffing, or touching it with its forepaws. Recognition memory was assessed using the discrimination index (discrimination index $=$ (novel familiar/novel + familiar)), corresponding to the difference between the time exploring the novel and the familiar object, corrected for total time exploring both objects. Two-way ANOVA was used to compare the differences between MAM and SAL groups, drug treatment, and the possible interaction between MAM and treatment on behavioral measures. Tukey's post hoc comparisons were conducted for significant main effects. All statistics were calculated using GraphPad Prism 8.

\section{Restraint stress}

Rats were restrained for $2 \mathrm{~h}$ in custom made Plexiglas cylinder restraint tubes (internal diameter $6 \mathrm{~cm}$ with length adjusted to rat size), placed in a ventilated transport tub. Rats in the control condition remained in their home cage for $2 \mathrm{~h}$. Rats were randomly assigned to receive vehicle $(1 \mathrm{ml} / \mathrm{kg})$ or POM $(3 \mathrm{mg} /$ $\mathrm{kg})$ i.p., $30 \mathrm{~min}$ prior to the stress or control condition. A subset of POM-treated rats was pretreated with LY34 (1 mg/kg, i.p.) $15 \mathrm{~min}$ prior to the POM injection. In vivo electrophysiological recordings of DA neuron activity were performed in anesthetized rats immediately following the restraint/control condition.

\section{RESULTS}

Pomaglumetad, i.p. dose-dependently reduces DA neuron activity in MAM rats

Electrophysiological recordings of DA neuron activity in the VTA were conducted from MAM rats and SAL rats that were pretreated with POM or VEH. VEH-treated MAM rats ( $n=7$ rats, 85 neurons) exhibited the anticipated elevation in population activity with an average of $1.8 \pm 0.1$ cells/track compared to VEH-treated SAL rats ( $n=6$ rats, 49 neurons), which had an average of $0.9 \pm 0.1$ cells/ track (Fig. 1a; two-way ANOVA MAM-by-POM interaction: $F(3,43)$ $=18.21, p<0.001$; post hoc MAM-vehicle vs SAL-vehicle: $p<$
$0.001)$. POM significantly reduced DA neuron population activity in MAM rats, at $1 \mathrm{mg} / \mathrm{kg}$ ( $n=6$ rats, 68 neurons; post hoc MAMvehicle vs MAM $1 \mathrm{mg} / \mathrm{kg}: p=0.019), 3 \mathrm{mg} / \mathrm{kg}(n=7$ rats, 61 neurons; post hoc MAM-vehicle vs MAM $3 \mathrm{mg} / \mathrm{kg}: p=0.007$ ) and $10 \mathrm{mg} / \mathrm{kg}$ ( $n=6$ rats, 38 neurons; post hoc MAM-vehicle vs MAM $10 \mathrm{mg} / \mathrm{kg}: p=0.001)$ compared to VEH-treated MAM rats. There was also a significant reduction in DA neuron population activity between $1 \mathrm{mg} / \mathrm{kg}$ and $10 \mathrm{mg} / \mathrm{kg}$ in MAM rats (post hoc MAM 1 $\mathrm{mg} / \mathrm{kg}$ vs MAM $10 \mathrm{mg} / \mathrm{kg}: p=0.003$ ). In contrast, there was no reduction in DA neuron population activity in POM-treated SAL rats, at $1 \mathrm{mg} / \mathrm{kg}$ ( $n=7$ rats, 46 neurons) or $3 \mathrm{mg} / \mathrm{kg}(n=6,48$ neurons), with a significant increase in population activity at 10 $\mathrm{mg} / \mathrm{kg}$ ( $n=6$ rats, 58 neurons; post hoc SAL-vehicle vs SAL $10 \mathrm{mg} /$ kg: $p=0.010$ ), compared to VEH-treated SAL rats (Fig. 1a). There was no significant change in firing rate (Fig. 1b) or bursting (Fig. 1c) with POM treatment compared to VEH treatment in MAM or SAL rats.

A separate cohort of MAM and SAL rats were treated with either acute or repeated POM or VEH (Fig. 1d; three-way ANOVA MAMby-POM interaction: $F(1,52)=11.82, p<0.001)$. Compared to their respective $\mathrm{VEH}$-treated group, POM-treated MAM rats showed a significant reduction in DA neuron population activity for both acute POM ( $n=6$ rats, 52 neurons; $p=0.002$ ) and repeated POM ( $n=9$ rats, 69 neurons; $p=0.011$ ). There was no significant difference between MAM rats treated acutely vs repeatedly with POM $(p>0.999)$ or MAM rats treated acutely vs repeatedly with VEH $(p=0.950)$. There were no significant differences in SAL rat DA neuron population activity across treatment and time groups (Fig. 1d). There were also no significant differences in firing rate (Fig. 1e) or bursting activity (Fig. 1f) of the DA neurons across all groups.

The effect of acute POM administration on DA neuron activity was examined in female MAM (Fig. 1g; two-way ANOVA main effects: for POM $F(1,26)=12.64, p=0.001$; for Estrous $F(1,26)=$ $9.213, p=0.005$, no interaction $F(1,26)=1.389, p=0.249)$ and SAL rats split into proestrous/estrous or metestrous/diestrous. Female MAM rats in proestrous/estrous treated with VEH ( $n=7$ rats, 79 neurons) displayed significantly greater DA neuron population activity compared to female MAM rats in metestrous/diestrous (Fig. $1 \mathrm{~h} ; n=7$ rats, 53 neurons; $p=0.037$ ). MAM rats in proestrous/ estrous treated with POM ( $n=8$ rats, 48 neurons) displayed significantly fewer spontaneously active DA neurons compared to MAM rats in proestrous/estrous treated with VEH (Fig. 1h; $p=$ $0.012)$. MAM rats in metestrous/diestrous treated with POM $(n=8$ rats, 40 neurons) did not display a significant difference in DA neuron population activity compared to VEH (Fig. $1 \mathrm{~h} ; p=0.354$ ). Female SAL rats displayed a main effect of estrous stage (Fig. 1i; two-way ANOVA main effects: for Estrous $F(1,25)=8.411, p=$ $0.008)$, but no main effect of POM treatment $(F(1,25)=0.357, p=$ 0.556 ) between SAL rats in proestrous/estrous treated with VEH ( $n$ $=7$ rats, 67 neurons) or POM ( $n=7$ rats, 60 neurons) and SAL rats in metestrous/diestrous treated with VEH ( $n=7$ rats, 30 neurons) or POM ( $n=8$ rats, 47 neurons) and no MAM-by-POM interaction $(F(1,25)=0.0518, p=0.822)$. There were no significant differences in firing rate or bursting activity of DA neurons in female rats across conditions.

Intra-ventral hippocampal infusion of pomaglumetad is sufficient to reduce DA neuron activity in MAM rats

Electrophysiological recordings of DA neuron activity in the VTA were conducted following infusion of either POM or VEH in the vHPC of MAM and SAL rats (Fig. 2a). MAM rats that received a VEH infusion ( $n=7$ rats, 75 neurons) displayed significantly higher DA neuron population activity compared to VEH-treated SAL rats (Fig. $2 \mathrm{~b} ; n=7$ rats, 51 neurons; two-way ANOVA main effects: for MAM, $F(1,26)=19.16, p=0.002$; for POM, $F(1,26)=13.17, p=$ 0.001 ; no interaction, $F(1,26)=3.405, p=0.0764$; post hoc MAM VEH Vs SAL VEH: $p=0.001)$. MAM rats that received a POM 
A.

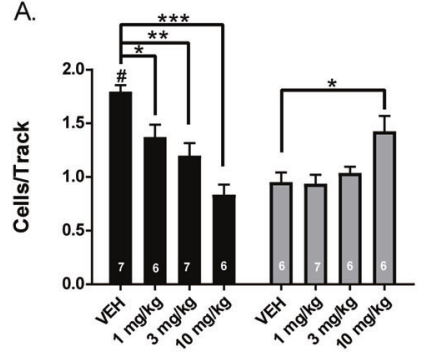

D.

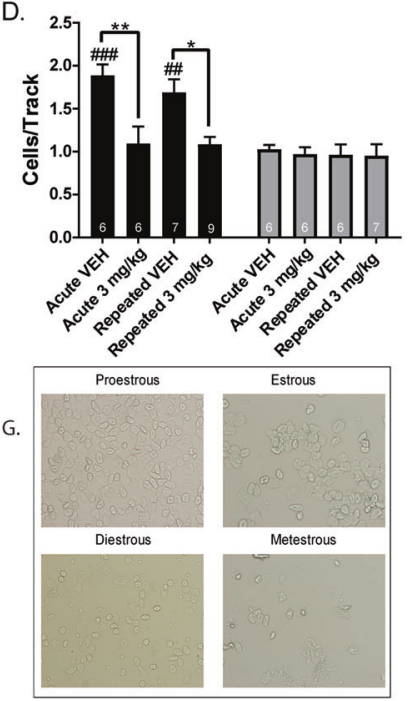

B.

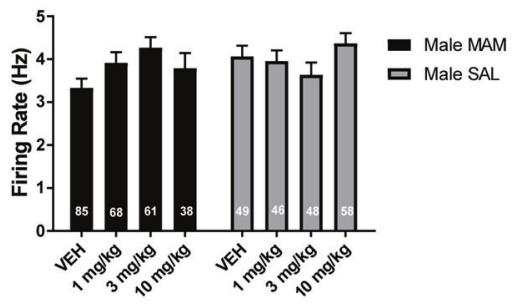

- Male MAM
口 Male SAL

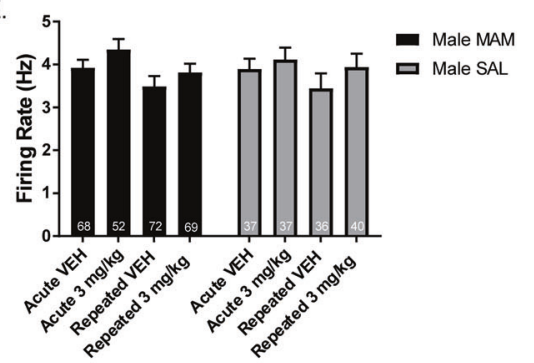

C.
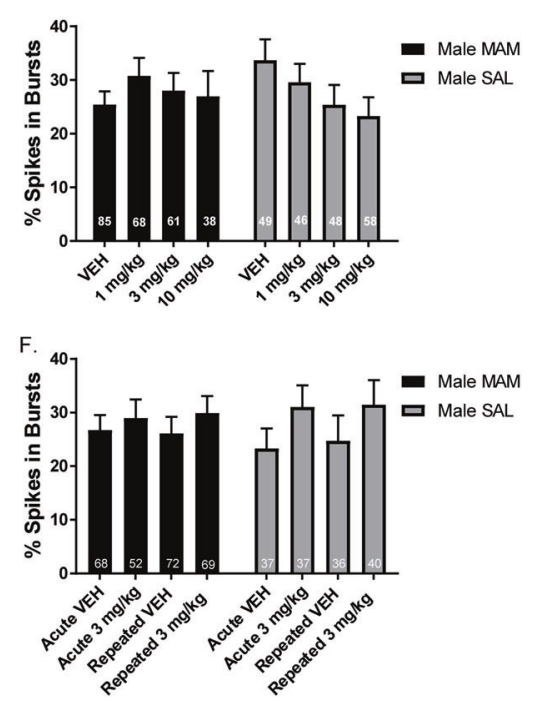

H.

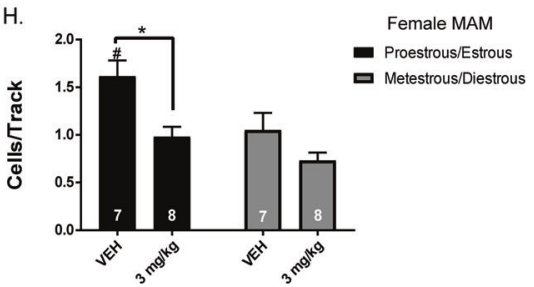

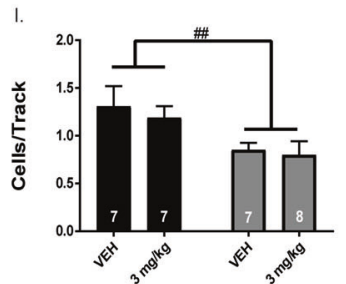

Female SAL - Proestrous/Estrous

Fig. 1 Pomaglumetad dose-dependently reduces DA neuron population activity in MAM rats. a In all, 30 min following POM i.p., MAM rats displayed a dose-dependent reduction in the number of spontaneously active DA neurons in the VTA compared to MAM rats that received VEH, which was not observed in SAL rats. There were no significant differences in the firing rate (b) or percentage of spikes in bursts (c) between POM and VEH administration in MAM or SAL rats. $\mathbf{d}$ There was no tolerance in the reduction in DA neuron population activity following $14 \mathrm{~d}$ repeated POM treatment in MAM rats, compared to acute treatment. There were no significant differences in the firing rate (e) or percentage of spikes in bursts (f) following either acute or repeated treatment in MAM or SAL rats. g Example pictures of proestrous (high density of nucleated cells and cornified cells), estrous (high density of cornified cells with leukocytes), metestrous (low density of cell debris, few cornified cells) and diestrous (low density of leukocytes and nucleated cells) (h) MAM rats displayed higher DA neuron population activity in proestrous/estrous compared to diestrous/metestrous. In all, 30 min following POM i.p., MAM rats in proestrous/estrous displayed a reduction in DA neuron population activity (i). SAL rats displayed higher DA neuron population activity in proestrous/estrous compared to

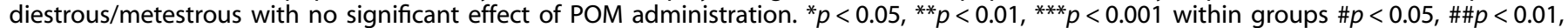
$\# \# \# p<0.001$ between groups.

infusion ( $n=9$ rats, 72 neurons) displayed a significant reduction in DA neuron population activity compared to vehicle-treated MAM rats $(p=0.002)$. POM treatment did not significantly impact DA neuron activity in SAL rats (Fig. 2b). There were no significant differences in the firing rate of DA neurons across groups (Fig. 2c), although there was a significant main effect of POM on percentage of spikes in burst (Fig. 2d; $F(1,224)=5.191, p=$ $0.024)$, without main effect of MAM $(F(1,224)=0.2483, p=0.619)$ or interaction $(F(1,224)=1.069, p=0.302)$.

\section{Pomaglumetad, i.p. increases novel object recognition in MAM}

\section{rats}

MAM rats pretreated with VEH displayed reduced interaction with a novel object compared to a familiar object, as measured by a discrimination index ((novel-familiar)/(novel+familiar)), compared to SAL rats treated with VEH (Fig. 3a; two-way ANOVA main effects: for MAM: $F(2,48)=8.505, p=0.005$, for MAM-by-POM interaction: $F(2,48)=4.188, p<0.021$; post hoc MAM VEH vs SAL VEH: $p=0.003$ ). MAM rats treated with $1 \mathrm{mg} / \mathrm{kg}$ POM did not improve significantly on the NOR compared to VEH, but MAM rats treated with $3 \mathrm{mg} / \mathrm{kg}$ showed a significant increase in discrimination index compared to VEH-treated MAM rats $(p=0.035)$. There was no significant effect of POM on discrimination index in SAL rats (Fig. 3a). MAM rats treated with $3 \mathrm{mg} / \mathrm{kg}$ POM displayed a significant reduction in time exploring either object in the test phase compared to VEH-treated SAL rats (Fig. 3b; two-way ANOVA main effect for POM: $F(2,48)=9.118, p<0.001$, no main effect of MAM $(F(1,48)=0.197, p=0.889)$ or interaction $(F(2,48)=1.715$, $p=0.191)$; post hoc SAL VEH vs SAL $3 \mathrm{mg} / \mathrm{kg}: p=0.003)$.

Pomaglumetad, i.p. blocks restraint stress-induced increase in DA neuron activity

Rats that were treated with VEH prior to acute restraint stress ( $n=$ 6 rats, 88 neurons) displayed a significant increase in DA neuron activity in the VTA in electrophysiological recordings performed immediately following 2 hour restraint stress (Fig. 4a; two-way ANOVA main effects: for Treatment, $F(2,30)=8.799, p=0.001$; for Stress, $F(1,30)=35.360, p<0.001$; for Treatment-by-Stress interaction: $F(2,30)=5.765, p<0.008$; post hoc VEH stress vs VEH control: $p<0.001)$, compared to rats that were treated with VEH and left in their home cage ( $n=6$ rats, 57 neurons). Rats that were treated with POM prior to acute restraint stress ( $n=6$ rats, 50 neurons) displayed a significant reduction in DA neuron population activity compared to stressed rats that were treated with VEH $(p<0.001)$. The effect of POM on DA neuron population activity in stressed rats was blocked by pretreatment with the mGluR2/3 antagonist LY34 ( $n=6$ rats, 64 neurons; $p=0.02$ ). Rats that were stressed that received both $\mathrm{POM}+\mathrm{LY} 34$ did not display a significant difference 

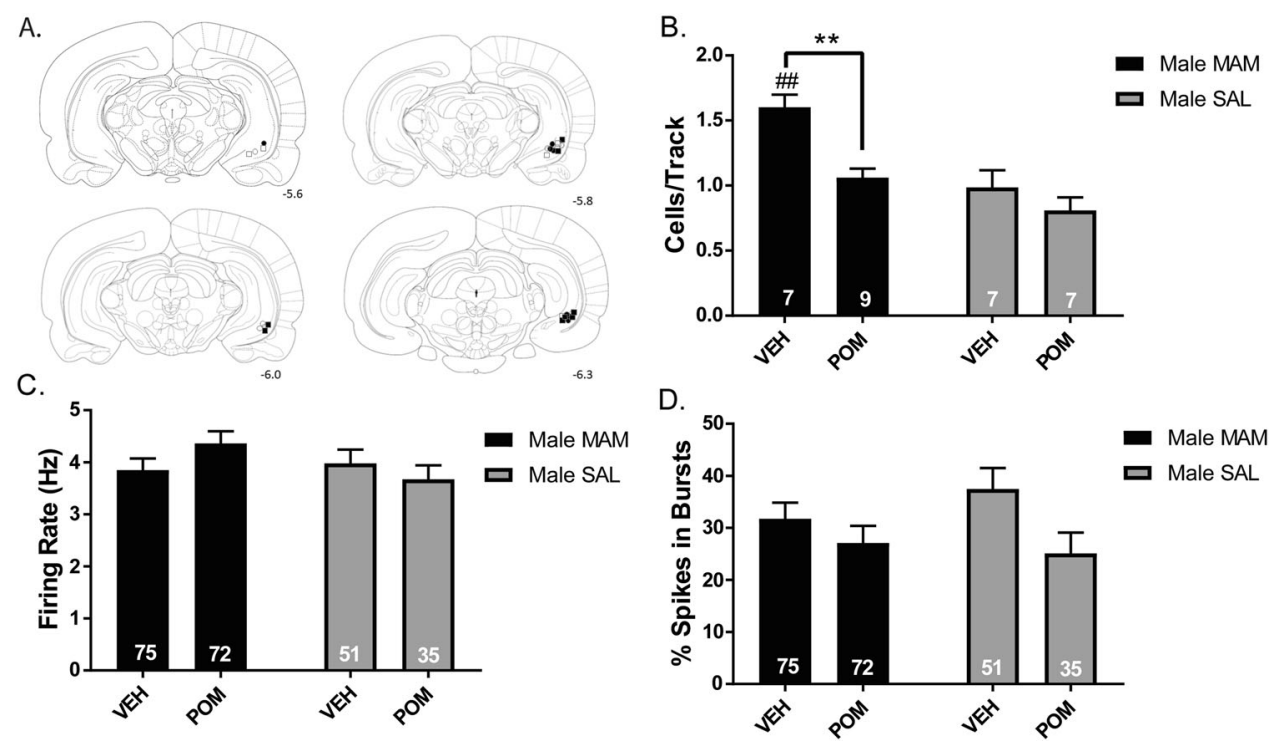

Fig. 2 Intra-ventral hippocampal infusion of pomaglumetad is sufficient to normalize DA neuron activity in MAM rats. a Placements of cannula for vHPC infusion for $\square$ MAM-VEH, MAM-POM, O SAL-VEH, SAL-POM. b Infusion of POM in the vHPC was sufficient to reduce DA neuron population activity in MAM rats, but did not impact SAL rats. $\mathbf{c}$, $\mathbf{d}$ No effect of intra-vHPC POM infusion on firing rate or the percentage

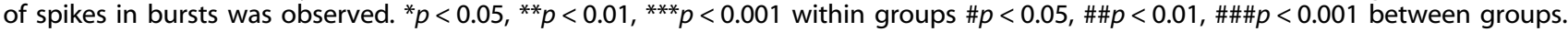
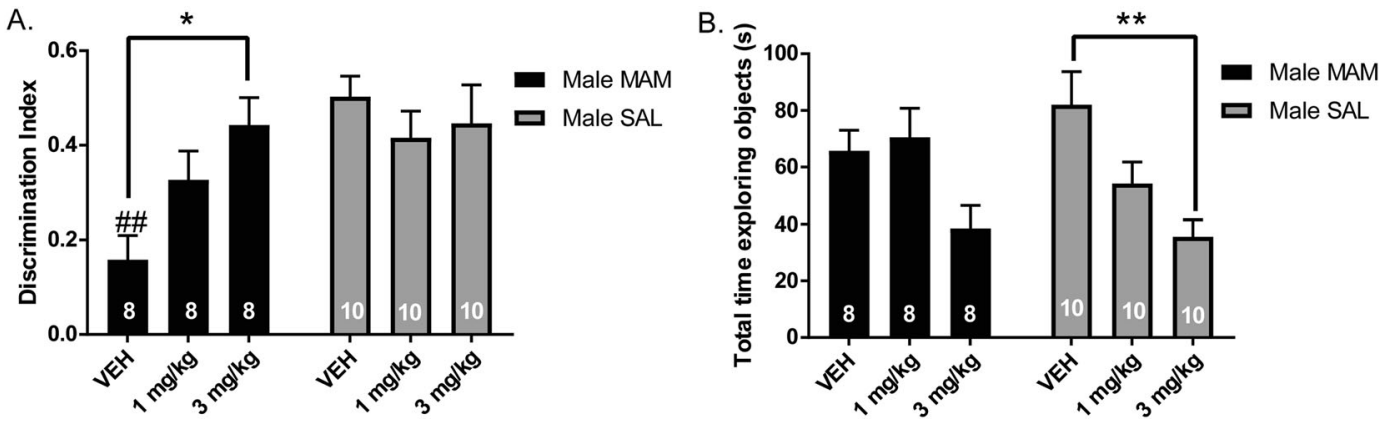

Fig. 3 Pomaglumetad increases novel object recognition in MAM rats. a MAM rats treated with VEH i.p. spent less time interacting with a novel object compared to a familiar object as measured by a discrimination index ((novel-familiar)/(novel + familiar)) compared to SAL rats. MAM rats treated with $3 \mathrm{mg} / \mathrm{kg}$, i.p. POM spent more time investigating the novel object compared to VEH treated MAM rats. There was no effect of POM on discrimination index in SAL rats. b POM reduced locomotor activity, measured by total time exploring objects in the test phase, which was significantly reduced in SAL rats treated with $3 \mathrm{mg} / \mathrm{kg}$ POM, i.p., compared to VEH-treated SAL rats. ${ }^{*} p<0.05,{ }^{* *} p<0.01$,

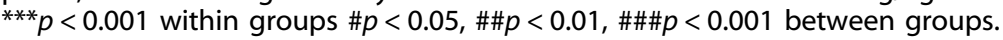
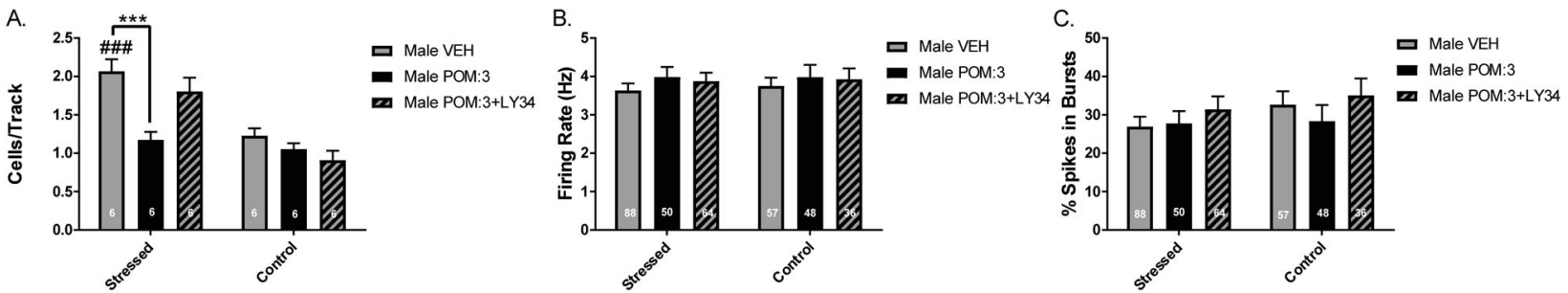

Fig. 4 Pomaglumetad blocks restraint stress-induced increase in DA neuron population activity. a Normal rats exposed to $2 \mathrm{~h}$ acute restraint stress following VEH pretreatment displayed increased DA neuron population activity compared to VEH-treated rats left in their home cage for $2 \mathrm{~h}$. POM pretreatment $(3 \mathrm{mg} / \mathrm{kg}$, i.p.) normalized DA neuron population activity in rats that received restraint stress to control levels, which was not observed in rats that received both POM and the mGluR2/3 antagonist LY34 prior to restraint stress. b, c No significant changes were observed in DA neuron firing rate or percentage of spikes in bursts. ${ }^{*} p<0.05,{ }^{* *} p<0.01,{ }^{* *} p<0.001$ within groups $\# p<0.05$, $\# \# p<0.01$, \#\#\#p<0.001 between groups.

in DA neuron population activity compared to stressed rats that were treated with VEH $(p=0.694)$. There was no significant effect of treatment on DA neuron activity in rats that were kept in their home cage (Fig. 4a). There were also no significant effects in firing rate (Fig. $3 \mathrm{~b}$ ) or the percentage of spikes in burst (Fig. 4c).

\section{DISCUSSION}

mGluR2/3 agonist normalizes dopamine neuron population activity in MAM rats

Our work demonstrates that the mGluR2/3 agonist, POM, decreases VTA DA neuron population activity in MAM rats via 
action in the vHPC. Previous studies that have examined the effects of mGluR2/3-targeting drugs on DA release have produced conflicting results. Some have shown that mGluR2/3 agonists do not affect striatal DA release at baseline [28, 31], which is consistent with the lack of effect we observed, in general, on DA neuron population activity in SAL rats and wild type rats in baseline conditions. Others have found that mGluR2/3 agonists can reduce striatal DA release [56], and that $m G l u R 2 / 3$ antagonist administration can increase striatal DA release at baseline [57]. In contrast, others have found that mGluR2/3 agonist administration produces an increase in striatal DA release at basal conditions $[58,59]$. Differing effects may relate to differences in microdialysis procedure, the potency and specificity of the drug used, or drug dose. Although mGluR2/3 are primarily located presynaptically on glutamatergic neurons to negatively regulate excess glutamate release, there are also receptors located postsynaptically and on glial cells [19], which can make dose an important factor [60]. This may explain the observed significant increase in DA neuron population activity in SAL rats at the highest dose tested $(10 \mathrm{mg} /$ $\mathrm{kg}$ ). Overall, our results indicate that $\mathrm{mGluR2} / 3$ agonists have the potential to reduce DA release through regulation of DA neuron population activity. However, this was only observed in states of increased DA neuron population activity driven indirectly by increased glutamate release from the vHPC in both MAM and restraint stress rats.

We also observed a significant reduction in DA neuron population activity in POM-treated female MAM rats, but the effect was dependent on estrous cycle stage. In both MAM and SAL rats, we observed differences in DA neuron population activity across the estrous cycle. In proestrous/estrous, and particularly in late proestrous/early estrous, we observed a significant increase in DA neuron population activity in MAM and SAL females compared to population activity in metestrous/diestrous. This is consistent with results observed by others in female MAM rats [61] and changes in DA release and DA-dependent behavior observed in wild type female rats across the estrous cycle $[62,63]$. Interestingly, POM only significantly reduced the increased DA neuron population activity during proestrous/estrous in MAM rats. One possible explanation is that increases and decreases in VHPC activity across the estrous cycle [64] may make the HPC more or less responsive to the effects of POM respectively in MAM rats. However, it remains unclear why POM did not have an effect on elevated DA neuron population activity in SAL females in proestrous/estrous. It is possible that vHPC activity was not sufficiently increased to observe a significant effect. Alternatively, interactions have been reported between estrogen receptors and mGluR2/3 receptors [65] that may differ between MAM and SAL rats.

We have previously shown that an $a 5 G A B A_{A}$ positive allosteric modulator (PAM) reduces the number of spontaneously active DA neurons in the VTA of MAM rats when administered systemically or when infused directly in the vHPC [66]. mGluR2/3 and a5GABA are both potential therapeutic targets to normalize vHPC output by reducing pyramidal neuron activity and increasing GABA receptor function, respectively. Similar to $m G l u R 2 / 3, a 5 G A B A_{A}$ is expressed in cortical and limbic regions, including the prefrontal cortex, amygdala, and HPC [67], and thus may be able to normalize excitatory-inhibitory activity across circuits relevant to schizophrenia [68]. However, withdrawal from prior chronic $D_{2}$ antagonist treatment prevented the ability of an a5GABA $A_{A}$ PAM to reduce amphetamine-induced hyperlocomotion in MAM rats [69]. Persistent DA supersensitivity following brief withdrawal from chronic $D_{2}$ antagonist treatment may similarly be a confound for the effects of POM on regulating DA neuron activity and may have contributed to its failure in clinical trials.

Effects of mGluR2/3 agonists beyond the hippocampus Intra-vHPC infusion of POM was sufficient to normalize DA neuron population activity in MAM rats. The effects of POM were not tested in the NAc, but due to the glutamatergic projections that the NAc receives from the vSub [70] there is a reasonable chance that an intra-NAc infusion of POM would produce a similar effect. The ventral pallidum (VP) holds a proportion of DA neurons in an inhibited state, such that reduced inhibition of VP from the NAc would reduce the number of spontaneously active dopamine neurons to a baseline state $[70,71]$. We hypothesize that regulation of excess glutamate anywhere within the vHPC-NAcVP circuit that regulates DA neuron population activity may be sufficient to affect DA neuron activity.

Although the present study focused on the action of POM in the VHPC, following systemic treatment POM would act in all brain regions that express mGluR2/3 receptors. mGluR2/3 receptors are located in numerous cortical and limbic brain regions, including the PFC and the amygdala [32-34]. POM's regulation of glutamate release in these regions would have widespread effects on related circuits and behavior, and such circuit effects have the potential to affect other symptoms of schizophrenia.

Behavioral tests of $\mathrm{mGluR} 2 / 3$ action

We found that pretreatment with POM dose-dependently improved the performance of MAM rats in NOR. We observed improvement in NOR despite the acute, dose-dependent decrease in time spent exploring the objects, which corresponded with previously reported dose-dependent reductions in spontaneous locomotor activity following POM administration [23]. The reduction in locomotor activity, as indirectly measured by total time exploring objects, only remained significant in the 2 nd trial of $S A L$ rats treated with the $3 \mathrm{mg} / \mathrm{kg}$ dose. Alterations in spontaneous locomotor behavior should be taken into consideration when interpreting locomotor-based behaviors, such as amphetamine-induced hyperlocomotion. POM may reduce movement regardless of effects on DA release. Thus, it effects on baseline locomotor activity may confound behavioral results and thus must be accounted for by normalizing the data to baseline.

Prevention of stress-induced increase in DA neuron activity The effects of POM on normalizing increased DA neuron population activity were not specific to the MAM model, but also observed in wild type rats exposed to restraint stress. It has been shown previously that acute restraint stress increases vHPC activity, which then drives increased DA neuron population activity [46]. Our results replicated this finding and, accordingly, pretreatment with POM prevented the restraint stress-induced increase in DA neuron activity. POM has similarly been found to reduce increased vHPC activity in an elevated plus maze task [43]. POM's reduction of VHPC activity and DA neuron population activity following stress may contribute to its anxiolytic-like effects found in other studies [43, 72-75]. Stress is proposed to play a critical role in the development of schizophrenia [76, 77] and early-life stress, in particular, can have a long-lasting impact on the DA system in adulthood [44, 78]. We have previously shown that peripubertal administration of the benzodiazepine, diazepam, can prevent the development of a number of MAM phenotypes in adulthood [79-81]. It is possible that other drugs with anxiolyticlike properties, such as mGluR2/3 agonists, may be similarly effective in reducing the long-lasting impact of stress during adolescence on DA neuron activity.

Overall, our results show that POM can normalize DA neuron population activity in conditions of either transient or chronic pathological increased HPC activity, and that its direct action in the VHPC is sufficient to produce this effect. However, it is important to note that our results do not indicate that its therapeutic action is identical to $\mathrm{D}_{2}$-targeting APDs or that its potential therapeutic benefits are limited to treating symptoms related to DA system dysregulation. For example, some patients who are treatment resistant do not show increased striatal DA synthesis capacity or release, but do demonstrate elevated 
glutamate levels in the anterior cingulate $[82,83]$. Therefore, patients who do not respond to $D_{2}$-targeting APDs may benefit from a drug that regulates excitatory-inhibitory imbalance. Better understanding of how mGluR2/3 agonists function mechanistically, particularly in clinically relevant states, may provide better insight into their potential as a therapeutic target.

\section{FUNDING AND DISCLOSURES}

This work was supported by NIH grant MH57440 (to AAG). SFS declares no conflicts of interest. AAG has received consulting fees from Alkermes, Lundbeck, Takeda, Roche, Lyra, Concert and funding from Lundbeck. The authors declare no competing interests.

\section{ACKNOWLEDGEMENTS}

We thank Niki MacMurdo and Christy Smolak for their technical assistance.

\section{AUTHOR CONTRIBUTIONS}

SFS designed the experiments, collected and analyzed data, interpreted data, and wrote the paper. AAG designed the experiments, interpreted data, and wrote the paper.

\section{ADDITIONAL INFORMATION}

Publisher's note Springer Nature remains neutral with regard to jurisdictional claims in published maps and institutional affiliations.

\section{REFERENCES}

1. Kapur S, Mamo D. Half a century of antipsychotics and still a central role for dopamine D 2 receptors. Prog Neuro-Psychopharmacol Biol Psychiatry. 2003;27:1081-90.

2. Creese I, Burt DR, Snyder SH. Dopamine receptor binding predicts clinical and pharmacological potencies of antischizophrenic drugs. Science. 1976;192:481-3.

3. Kapur S, et al. Relationship between dopamine D2 occupancy, clinical response, and side effects: a double-blind PET study of first-episode schizophrenia. Am J Psychiatry. 2000;157:514-20.

4. Burris K, et al. Aripiprazole, a novel antipsychotic, is a high-affinity partial agonist at human dopamine D2 receptors. The. J Pharmacol Exp therapeutics. 2002;302:381-9.

5. Grace A, Bunney B. Induction of depolarization block in midbrain dopamine neurons by repeated administration of haloperidol: analysis using in vivo intracellular recording. J Pharmacol Exp Therapeutics. 1986;238:1092-1100.

6. Valenti $O$, et al. Antipsychotic drugs rapidly induce dopamine neuron depolarization block in a developmental rat model of schizophrenia. J Neurosci. 2011;31:12330-8.

7. Sonnenschein SF, Gill KM, Grace AA. State-dependent effects of the D 2 partial agonist aripiprazole on dopamine neuron activity in the MAM neurodevelopmental model of schizophrenia. Neuropsychopharmacology. 2019;44:572

8. Howes $\mathrm{O}$, et al. Progressive increase in striatal dopamine synthesis capacity as patients develop psychosis: a PET study. Mol psychiatry. 2011;16:885.

9. Laruelle M, Abi-Dargham A. Dopamine as the wind of the psychotic fire: new evidence from brain imaging studies. J Psychopharmacol. 1999;13:358-71.

10. Leucht $\mathrm{S}$, et al. Comparative efficacy and tolerability of 15 antipsychotic drugs in schizophrenia: a multiple-treatments meta-analysis. Lancet. 2013;382:951-62.

11. Saha S, et al. A systematic review of the prevalence of schizophrenia. PLoS Med. 2005;2:e141.

12. Kane J, et al. Clozapine for the treatment-resistant schizophrenic: a double-blind comparison with chlorpromazine. Arch Gen psychiatry. 1988;45:789-96.

13. Lieberman JA, et al. Clinical effects of clozapine in chronic schizophrenia: response to treatment and predictors of outcome. Am J Psychiatry. 1994;151:1744-52.

14. Remington $\mathrm{G}$, et al. Augmentation strategies in clozapine-resistant schizophrenia. CNS drugs. 2005;19:843-72.

15. Lieberman JA, et al. Effectiveness of antipsychotic drugs in patients with chronic schizophrenia. N. Engl j Med. 2005;2005:1209-23.

16. Ho B-C, et al. Long-term antipsychotic treatment and brain volumes: a longitudinal study of first-episode schizophrenia. Arch Gen psychiatry. 2011;68:128-37
17. Silvestri $S$, et al. Increased dopamine D2 receptor binding after long-term treatment with antipsychotics in humans: a clinical PET study. Psychopharmacology. 2000;152:174-80.

18. Lidow MS, Elsworth JD, Goldman-Rakic PS. Down-regulation of the D1 and D5 dopamine receptors in the primate prefrontal cortex by chronic treatment with antipsychotic drugs. J Pharmacol Exp Therapeutics. 1997;281:597-603.

19. Nicoletti $F$, et al. Metabotropic glutamate receptors: from the workbench to the bedside. Neuropharmacology. 2011;60:1017-41.

20. Benes FM, Berretta S. GABAergic interneurons: implications for understanding schizophrenia and bipolar disorder. Neuropsychopharmacology. 2001;25:1-27.

21. Lewis DA, Hashimoto T, Volk DW. Cortical inhibitory neurons and schizophrenia. Nat Rev Neurosci. 2005;6:312-24

22. Lodge DJ, Grace AA. Hippocampal dysregulation of dopamine system function and the pathophysiology of schizophrenia. Trends Pharmacol Sci. 2011;32:507-13.

23. Rorick-Kehn LM, et al. In vivo pharmacological characterization of the structurally novel, potent, selective mGlu2/3 receptor agonist LY404039 in animal models of psychiatric disorders. Psychopharmacology. 2007;193:121-36.

24. Mezler M, et al. LY-2140023, a prodrug of the group II metabotropic glutamate receptor agonist LY-404039 for the potential treatment of schizophrenia. Curr Opin Investig Drugs. 2010;11:833-45.

25. Adams $\mathrm{DH}$, et al. A long-term, phase 2, multicenter, randomized, open-label, comparative safety study of pomaglumetad methionil (LY2140023 monohydrate) versus atypical antipsychotic standard of care in patients with schizophrenia. BMC psychiatry. 2013;13:143.

26. Marek G. When is a Proof-of-Concept (POC) not a POC? Pomaglumetad (LY2140023) as a Case Study for Antipsychotic Efficacy. Curr Pharm Des. 2015;21:3788-96.

27. Kinon BJ, et al. Exploratory Analysis for a Targeted Patient Population Responsive to the Metabotropic Glutamate 2/3 Receptor Agonist Pomaglumetad Methionil in Schizophrenia. Biol Psychiatry. 2015;78:754-62.

28. Moghaddam B, Adams BW. Reversal of phencyclidine effects by a group II metabotropic glutamate receptor agonist in rats. Science. 1998;281:1349-52.

29. Lorrain $D$, et al. Effects of ketamine and n-methyl-d-aspartate on glutamate and dopamine release in the rat prefrontal cortex: modulation by a group II selective metabotropic glutamate receptor agonist LY379268. Neuroscience. 2003:3:697-706.

30. Homayoun $\mathrm{H}$, Moghaddam B. NMDA receptor hypofunction produces opposite effects on prefrontal cortex interneurons and pyramidal neurons. J Neurosci. 2007;27:11496-11500.

31. Pehrson AL, Moghaddam B. Impact of metabotropic glutamate $2 / 3$ receptor stimulation on activated dopamine release and locomotion. Psychopharmacology. 2010;211:443-55.

32. Neki $A$, et al. Pre-and postsynaptic localization of a metabotropic glutamate receptor, mGluR2, in the rat brain: an immunohistochemical study with a monoclonal antibody. Neurosci Lett. 1996;202:197-200.

33. Ohishi $\mathrm{H}$, et al. Distribution of the messenger RNA for a metabotropic glutamate receptor, mGluR2, in the central nervous system of the rat. Neuroscience. 1993;53:1009-18.

34. Ohishi $\mathrm{H}$, et al. Distribution of the mRNA for a metabotropic glutamate receptor (mGluR3) in the rat brain: an in situ hybridization study. J Comp Neurol. 1993;335:252-66.

35. Blümcke I, et al. Immunohistochemical distribution of metabotropic glutamate receptor subtypes mGluR1b, mGluR2/3, mGluR4a and mGluR5 in human hippocampus. Brain Res. 1996;736:217-26.

36. Heckers S, Konradi C. GABAergic mechanisms of hippocampal hyperactivity in schizophrenia. Schizophrenia Res. 2015;167:4-11.

37. Konradi $C$, et al. Hippocampal interneurons are abnormal in schizophrenia. Schizophrenia Res. 2011;131:165-73.

38. Lodge DJ, Behrens MM, Grace AA. A loss of parvalbumin-containing interneurons is associated with diminished oscillatory activity in an animal model of schizophrenia. J Neurosci. 2009;29:2344-54.

39. Lodge DJ, Grace AA. Aberrant hippocampal activity underlies the dopamine dysregulation in an animal model of schizophrenia. J Neurosci. 2007;27:11424-30.

40. Modinos G, et al. Translating the MAM model of psychosis to humans. Trends Neurosci. 2015;38:129-38.

41. Howes OD, et al. The nature of dopamine dysfunction in schizophrenia and what this means for treatment: meta-analysis of imaging studies. Arch Gen psychiatry. 2012;69:776-86.

42. McCutcheon RA, Abi-Dargham A, Howes OD. Schizophrenia, dopamine and the striatum: from biology to symptoms. Trends Neurosci. 2019;42:205-220

43. Linden A, et al. Anxiolytic activity of the MGLU2/3 receptor agonist LY354740 on the elevated plus maze is associated with the suppression of stress-induced c-Fos in the hippocampus and increases in c-Fos induction in several other stresssensitive brain regions. Neuropsychopharmacology. 2004;29:502. 
44. Gomes FV, Grace AA. Prefrontal cortex dysfunction increases susceptibility to schizophrenia-like changes induced by adolescent stress exposure. Schizophrenia Bull. 2016;43:592-600.

45. Hammond RS, Tull LE, Stackman RW. On the delay-dependent involvement of the hippocampus in object recognition memory. Neurobiol Learn Mem. 2004;82:26-34.

46. Valenti O, Lodge DJ, Grace AA. Aversive stimuli alter ventral tegmental area dopamine neuron activity via a common action in the ventral hippocampus. J Neurosci. 2011;31:4280-9.

47. Fell MJ, et al. Evidence for the role of metabotropic glutamate (mGlu) 2 not mGlu3 receptors in the preclinical antipsychotic pharmacology of the $\mathrm{mGlu2} / 3$ receptor agonist (-)-(1R, 4S, 5S, 6S)-4-Amino-2-sulfonylbicyclo [3.1. 0] hexane-4, 6dicarboxylic Acid (LY404039). J Pharmacol Exp Therapeutics. 2008;326:209-17.

48. Lu L, et al. Systemic and central amygdala injections of the mGluR2/3 agonist LY379268 attenuate the expression of incubation of cocaine craving. Biol psychiatry. 2007;61:591-8.

49. Myal S, O'Donnell $P$, Counotte DS. Nucleus accumbens injections of the mGluR2/3 agonist LY379268 increase cue-induced sucrose seeking following adult, but not adolescent sucrose self-administration. Neuroscience. 2015;305:309-15.

50. Marcondes F, Bianchi F, Tanno A. Determination of the estrous cycle phases of rats: some helpful considerations. Braz J Biol. 2002;62(4A):609-14.

51. Hubscher $C$, Brooks D, Johnson J. A quantitative method for assessing stages of the rat estrous cycle. Biotech Histochemistry. 2005;80:79-87.

52. Ungless MA, Grace AA. Are you or aren't you? Challenges associated with physiologically identifying dopamine neurons. Trends Neurosci. 2012;35:422-30.

53. Grace A, Bunney B. Intracellular and extracellular electrophysiology of nigral dopaminergic neurons-1. Identification and characterization. Neuroscience. 1983;10:301-15.

54. Grace AA, Bunney BS. The control of firing pattern in nigral dopamine neurons: burst firing. J Neurosci. 1984;4:2877-90.

55. Paxinos G, Watson C. The rat brain in stereotaxic coordinates. Qingchuan Zhuge translate. Vol 32. Beijing: People's Medical Publishing House; 2007.

56. Hu G, et al. The regulation of dopamine transmission by metabotropic glutamate receptors. J Pharmacol Exp Therapeutics. 1999;289:412-6.

57. Karasawa J-i, Yoshimizu T, Chaki S. A metabotropic glutamate 2/3 receptor antagonist, MGS0039, increases extracellular dopamine levels in the nucleus accumbens shell. Neurosci Lett. 2006;393:127-30.

58. Ohno $M$, Watanabe $S$. Persistent increase in dopamine release following activation of metabotropic glutamate receptors in the rat nucleus accumbens. Neurosci Lett. 1995;200:113-6.

59. Verma A, Moghaddam B. Regulation of striatal dopamine release by metabotropic glutamate receptors. Synapse. 1998;28:220-6.

60. Jin LE, et al. mGluR2/3 mechanisms in primate dorsolateral prefrontal cortex: evidence for both presynaptic and postsynaptic actions. Mol psychiatry. 2017;22:1615.

61. Perez SM, Chen L, Lodge DJ. Alterations in dopamine system function across the estrous cycle of the MAM rodent model of schizophrenia. Psychoneuroendocrinology. 2014;47:88-97.

62. Castner SA, Xiao L, Becker JB. Sex differences in striatal dopamine: in vivo microdialysis and behavioral studies. Brain Res. 1993;610:127-34.

63. Xiao L, Becker JB. Quantitative microdialysis determination of extracellular striatal dopamine concentration in male and female rats: effects of estrous cycle and gonadectomy. Neurosci Lett. 1994;180:155-8.

64. Scharfman HE, et al. Hippocampal excitability increases during the estrous cycle in the rat: a potential role for brain-derived neurotrophic factor. J Neurosci. 2003;23:11641-52.
65. Eisinger KRT, et al. Interactions between estrogen receptors and metabotropic glutamate receptors and their impact on drug addiction in females. Horm Behav. 2018;104:130-7.

66. Gill KM, et al. A novel a5GABAAR-positive allosteric modulator reverses hyperactivation of the dopamine system in the MAM model of schizophrenia. Neuropsychopharmacology. 2011;36:1903.

67. Lingford-Hughes $A$, et al. Imaging the GABA-benzodiazepine receptor subtype containing the a5-subunit in vivo with [11C] Ro15 4513 positron emission tomography. J Cereb Blood Flow Metab. 2002;22:878-89.

68. Gill K, Grace A. The role of a5 GABAA receptor agonists in the treatment of cognitive deficits in schizophrenia. Curr Pharm Des. 2014;20:5069-76.

69. Gill KM, et al. Prior antipsychotic drug treatment prevents response to novel antipsychotic agent in the methylazoxymethanol acetate model of schizophrenia. Schizophrenia Bull. 2014;40:341-50.

70. Floresco SB, Todd CL, Grace AA. Glutamatergic afferents from the hippocampus to the nucleus accumbens regulate activity of ventral tegmental area dopamine neurons. J Neurosci. 2001;21:4915-22.

71. Floresco SB, et al. Afferent modulation of dopamine neuron firing differentially regulates tonic and phasic dopamine transmission. Nat Neurosci. 2003;6:968.

72. Helton DR, et al. Anxiolytic and side-effect profile of LY354740: a potent, highly selective, orally active agonist for group II metabotropic glutamate receptors. J Pharmacol Exp Therapeutics. 1998;284:651-60.

73. Shekhar A, Keim S. LY354740, a potent group II metabotropic glutamate receptor agonist prevents lactate-induced panic-like response in panic-prone rats. Neuropharmacology. 2000;39:1139-46.

74. Kłodzińska A, et al. Potential anti-anxiety, anti-addictive effects of LY 354740, a selective group II glutamate metabotropic receptors agonist in animal models. Neuropharmacology. 1999;38:1831-9.

75. Spooren WP, et al. Pharmacological and endocrinological characterisation of stress-induced hyperthermia in singly housed mice using classical and candidate anxiolytics (LY314582, MPEP and NKP608). Eur J Pharmacol. 2002;435: 161-70.

76. Corcoran C, et al. The stress cascade and schizophrenia: etiology and onset Schizophrenia Bull. 2003;29:671-92.

77. Holtzman C, et al. Stress and neurodevelopmental processes in the emergence of psychosis. Neuroscience. 2013;249:172-91.

78. Gomes FV, Zhu X, Grace AA. The pathophysiological impact of stress on the dopamine system is dependent on the state of the critical period of vulnerability. Mol. Psychiatry. 2019: 1-14. https://doi.org/10.1038/s41380-019-0514-1.

79. Du Y, Grace AA. Peripubertal diazepam administration prevents the emergence of dopamine system hyperresponsivity in the MAM developmental disruption model of schizophrenia. Neuropsychopharmacology. 2013;38:1881.

80. Du Y, Grace AA. Loss of parvalbumin in the hippocampus of MAM schizophrenia model rats is attenuated by peripubertal diazepam. Int J Neuropsychopharmacol 2016;19:1-5.

81. Du Y, Grace AA. Amygdala hyperactivity in MAM model of schizophrenia is normalized by peripubertal diazepam administration. Neuropsychopharmacology. 2016;41:2455-62.

82. Mouchlianitis $\mathrm{E}$, et al. Treatment-resistant schizophrenia patients show elevated anterior cingulate cortex glutamate compared to treatment-responsive. Schizophrenia Bull. 2015;42:744-52.

83. Demjaha A, et al. Antipsychotic treatment resistance in schizophrenia associated with elevated glutamate levels but normal dopamine function. Biol Psychiatry. 2014;75:e11-3. 